\title{
Pseudo-progressive bone lesion in a relapsed infant leukemia after chimeric antigen reseptor T-cell therapy
}

\author{
Satomi Yokoyama ${ }^{1}$, Yuhki Koga ${ }^{2}$, Hiroshi Aasai ${ }^{2}$, Hiroaki Ono $^{2}$, Kensaku Kohrogi ${ }^{3}$, \\ Tadashi Anan ${ }^{3}$, Koji Kato ${ }^{2}$, and Shouichi Ohga ${ }^{4}$ \\ ${ }^{1}$ Kyushu University \\ ${ }^{2}$ Graduate School of Medical Sciences, Kyushu University \\ ${ }^{3}$ Kumamoto University \\ ${ }^{4}$ Graduate School of Medicial Sciences, Kyushu University
}

May 5, 2021

Title

Pseudo-progressive bone lesion in a relapsed infant leukemia after chimeric antigen reseptor T-cell therapy

Satomi Yokoyama ${ }^{1}$, Yuhki Koga ${ }^{1}$, Hiroshi Asai ${ }^{1}$, Hiroaki Ono ${ }^{1}$, Kensaku Kohrogi ${ }^{2}$, Tadashi Anan ${ }^{2}$, Koji Kato $^{3}$, Shouichi Ohga ${ }^{1}$

1. Department of Pediatrics, Graduate School of Medical Sciences, Kyushu University, 3-1-1 Maidashi, Higashi-ku, Fukuoka 812-8582, Japan.

2. Department of Pediatrics, Graduate School of Medical Sciences, Kumamoto University, 1-1-1 Honjyo, Thyuou-Ku, Kumamoto 860-8556, Japan.

3. Department of Medicine and Biosystemic Science, Graduate School of Medical Sciences, Kyushu University, 3-1-1 Maidashi, Higashi-ku, Fukuoka, 812-8582, Japan.

\section{Corresponding Author}

Yuhki Koga, MD, PhD

3-1-1, Maidashi, Higashi-ku, Fukuoka 812-8582, Japan

TEL: +81-92-642-5421

FAX: +81-92-642-5435

Mail to: koga.yuhki.743@m.kyushu-u.ac.jp

\section{Word count}

Main text: 500 words

The number of Table and Supplementary figure

Table: 1

Supplementary figure: 1

A Short Running Title: Pseudo-progression in ALL after CART therapy

Key words: Pseudo-progression, relapsed ALL, CAR T-cell therapy 


\section{Abbreviation}

\begin{tabular}{ll}
\hline CAR T-cells & chimeric antigen receptor redirected T cells \\
\hline ALL & acute lymphoblastic leukemia \\
EL & extramedullary lesion \\
CR & complete remission \\
BM & bone marrow \\
HLA & human leukocyte antigen \\
cGVHD & chronic graft-versus-host disease \\
CRS & cytokine release syndrome \\
HSCT & hematopoietic stem cell transplantation \\
\hline
\end{tabular}

To the Editor,

Chimeric antigen receptor redirected $\mathrm{T}$ cells (CAR-T cells) have enabled us to offer a promising treatment for relapsed or refractory acute lymphoblastic leukemia (ALL). ${ }^{1}$ However, there is little information about adverse events associated with the administration of CAR T-cells. ${ }^{2,3}$ The changes in extramedullary lesion (EL) of relapsed or refractory ALL after CAR T-cell therapy have been recognized only in a few cases. ${ }^{4,5}$ We report herewith pseudo-progression as an augmented immune response to the EL in a boy with relapsed infant ALL after CAR T-cell therapy.

A 10-year-old boy received Tisagenlecleucel because of intractable treatment course of ALL. This patient had received a diagnosis of infant ALL with $M L L-E N L$ fusion gene at age 4 months. Complete remission (CR) was obtained after the first induction therapy, but a bone marrow (BM) relapse occurred during the course of the salvage chemotherapy. At 14 months of age, he received umbilical cord blood transplantation from an unrelated human leukocyte antigen (HLA)-one locus mismatched donor that led to the second CR. He then underwent a haploidentical BM transplantation from his mother at age 2 because of the second $\mathrm{BM}$ relapse. The third $\mathrm{CR}$ was achieved after the last BM transplantation, but BM and testicular relapses of ALL occurred at age 7 years. Repeated infusions of mother's lymphocytes controlled the disease on the developing chronic graft-versus-host disease (cGVHD) until age 9 years, when EL including the left leg bone led to limping. Five courses of blinatumomab failed to control the progressive disease.

Tisagenlecleucel was administered in the non-remitting state following lymphodepleting chemotherapy with fludarabine and cyclophosphamide. Grade 2 cytokine release syndrome (CRS) developed with fever and dyspnea on day 3 , and then required tocilizumab for the appreciable control. Prior to CAR-T therapy, his left leg with the bone lesion started to be swollen. The painful leg size increased with heat after CRS occurred. The second dose tocilizumab on day 6 and methylprednisolone on day 7 led to a defervescence and resolution of the affected leg pain. The local inflammation improved by day 14 to the size of contralateral leg (Supplementary figure ). One month later, he obtained cellular and molecular CR without EL.

Pseudo-progression after immunotherapies have been described in malignant tumors including high-grade glioblastomas, non-small-cell lung carcinoma, and melanoma, but less commonly in hematological malignancies. Table 1 summarizes all reported cases of leukemia and lymphoma that presented pseudo-progression during CAR-T therapy. ${ }^{2-5}$ This patient showed a rapid enlargement of EL with heat and pain after administration of CAR-T cells. Clinical course and serum interleukin-6 dominant cytokine profile (data not shown ) along with the time course of CRS indicated a pseudo-progression but not true-progression of EL. The present patient received CAR-T cells after hematopoietic stem cell transplantation (HSCT). He had controlable cGVHD without immunosuppressants on mixed donor chimerism (mother 99.3\%) after repetitive HSCT at the time of CAR-T cell infusion. This condition might augment the immune response to leukemic cells. According to the CRS magnitude and EL sites, careful management are needed for pseudo-progression after CAR-T cell therapy. 


\section{CONFLICT OF INTEREST}

The authors declare that there are no conflicts of interest in association with this report.

\section{ACKNOWLEDGEMENTS}

The authors thank the patient and their family for participating in this report.

\section{REFERENCES}

1. Maude SL, Frey N, Shaw PA, et al. Chimeric Antigen Receptor T Cells for Sustained Remissions in Leukemia. N Engl J Med.2014;371:1507-1517.

2. Wang J, Hu Y, Yang S, et al. Role of Fluorodeoxyglucose Positron Emission Tomography/Computed Tomography in Predicting the Adverse Effects of Chimeric Antigen Receptor T Cell Therapy in Patients with Non-Hodgkin Lymphoma. Biol Blood Marrow Transplant.2019;25:1092-1098.

3. Danylesko I, Shouval R, Shem-Tov N, et al. Immune imitation of tumor progression after anti-CD19 chimeric antigen receptor $\mathrm{T}$ cells treatment in aggressive B-cell lymphoma. Bone Marrow Transplant. 2020;18- doi:10.1038/s41409-020-01156-y.

4. Denton CC, Gange WS, Abdel-Azim H, et al. Bilateral retinal detachment after chimeric antigen receptor T-cell therapy. Blood Adv. 2020;4:2158-2162.

5. Huang J, Rong L, Wang E, Fang Y. Pseudoprogression of extramedullary disease in relapsed acute lymphoblastic leukemia after CAR T-cell therapy. Immunotherapy 2021;13:5-10.

Figure legends

Supplementary figure A. Clinical presentation of the patient after the the

administration of CAR T-cells. B, C. MRI changes of the leg with pseudo-progression;

Before CAR-T treatment and After CAR-T treatment (one month after administration)

\section{Hosted file}

Tables Letter-pseudoPG CART 20210503 (2).pdf available at https://authorea.com/users/412139/ articles/520907-pseudo-progressive-bone-lesion-in-a-relapsed-infant-leukemia-afterchimeric-antigen-reseptor-t-cell-therapy

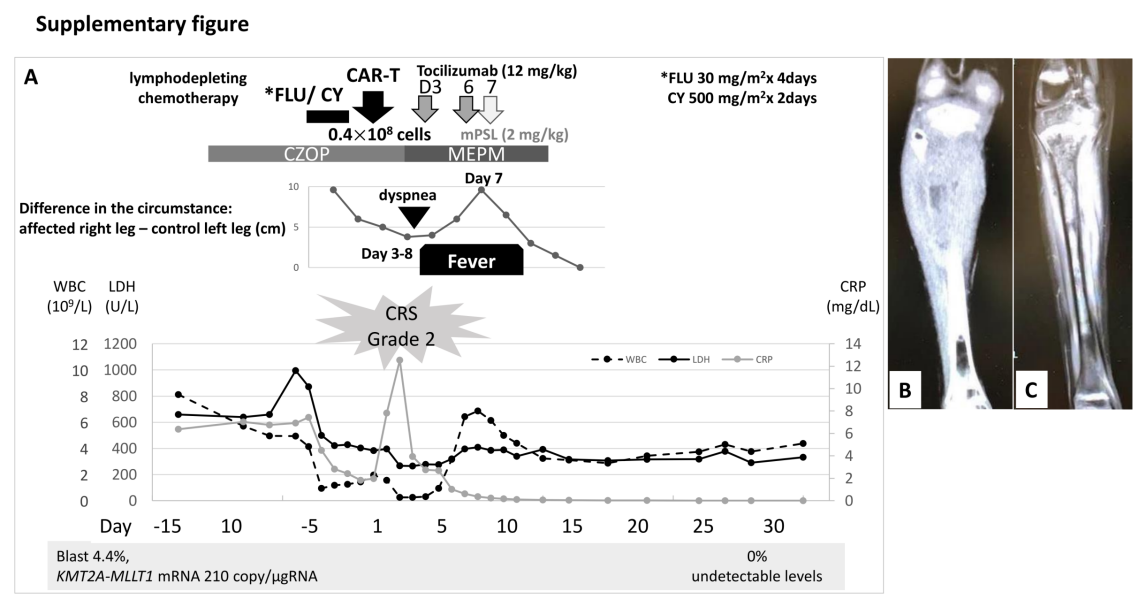

